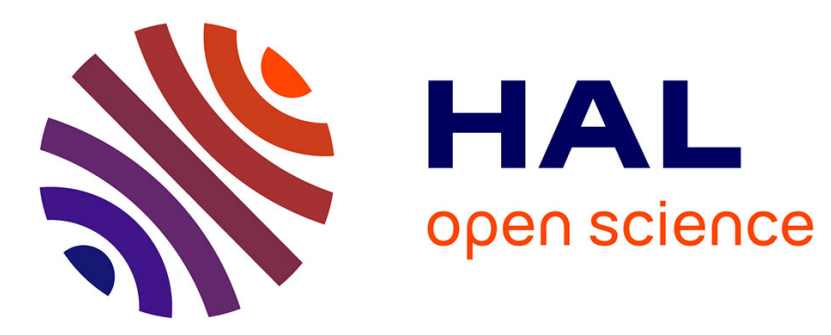

\title{
Difference equations, uniform quasiclassical asymptotics and Airy functions
}

\author{
A. Fedotov, F. Klopp
}

\section{To cite this version:}

A. Fedotov, F. Klopp. Difference equations, uniform quasiclassical asymptotics and Airy functions. International Conference "Days on Diffraction 2018", Jun 2018, St. Petersbourg, Russia. pp.98-101, 10.1109/DD.2018.8553493 . hal-02058815

\section{HAL Id: hal-02058815 https://hal.sorbonne-universite.fr/hal-02058815}

Submitted on 12 Mar 2019

HAL is a multi-disciplinary open access archive for the deposit and dissemination of scientific research documents, whether they are published or not. The documents may come from teaching and research institutions in France or abroad, or from public or private research centers.
L'archive ouverte pluridisciplinaire HAL, est destinée au dépôt et à la diffusion de documents scientifiques de niveau recherche, publiés ou non, émanant des établissements d'enseignement et de recherche français ou étrangers, des laboratoires publics ou privés. 


\title{
Difference equations, uniform quasiclassical asymptotics and Airy functions
}

\author{
A. Fedotov \\ Saint Petersburg State University, Universitetskaya nab. 7/9, St. Petersburg, 199034, Russia; \\ e-mail: a.fedotov@spbu.ru \\ F. Klopp \\ Sorbonne Universités, UPMC Univ. Paris 06, UMR 7586, IMJ-PRG, F-75005, Paris, France; Univ. \\ Paris Diderot, Sorbonne Paris Cité, UMR 7586, IMJ-PRG, F-75205 Paris, France; CNRS, UMR 7586, \\ IMJ-PRG, F-75005, Paris, France; e-mail: frederic.klopp@imj-prg.fr
}

\begin{abstract}
We consider the second order difference equation $\psi(z+h)+\psi(z-h)+v(z) \psi(z)=0$ where $z$ is a complex variable, $h>0$ is a parameter, and $v$ is an analytic function. As $h \rightarrow 0$ the analytic solutions to this equation have quasiclassical behavior. In this note we describe their uniform asymptotics in neighborhoods of simple turning points, the neighborhoods being independent of $h$.
\end{abstract}

\section{INTRODUCTION}

We study analytic solutions to the second order difference equation

$$
\psi(z+h)+\psi(z-h)+v(z) \psi(z)=0
$$

where $z$ is a complex variable, $h>0$ is a parameter, and $v$ is an analytic function. We describe their asymptotics as $h \rightarrow 0$.

Note that the parameter $h$ appears to be a standard quasiclassical parameter. Indeed, formally, $\psi(z+h)=\sum_{l=0}^{\infty} \frac{h^{l}}{l !} \frac{d^{l} \psi}{d z^{l}}(z)=e^{h \frac{d}{d z}} \psi(z)$, and $h$ can be regarded as a small parameter in front of the derivative.

Difference equations in the complex plane in the quasiclassical regime arise in many fields of mathematical physics. For example, they arise when studying an electron in a crystal placed in a constant magnetic field (e.g., [1, 2]), wave scattering by thin wedges (e.g., [3]), one-dimensional quasiperiodic differential Schrödinger equations with two frequencies, one being small with respect to the other (e.g., [4]). Similar problems arise in the theory of orthogonal polynomials (e.g., [5]).

The quasiclassical asymptotics of analytic solutions to ordinary differential equations in the complex plane are well-known (e.g., $[6,7])$. The method is often called complex WKB method. Its analog for difference equations was developed in $[8,9,10]$.
This note is devoted to uniform asymptotic formulas describing analytic solutions to (1) in neighborhoods of simple turning points (see the definition below), the neiborhoods being independent of $h$.

\section{A VERY SHORT INTRODUCTION TO THE COM- PLEX WKB METHOD}

Below, $U$ denotes the domain of analyticity of $v$.

\subsection{Complex momentum}

The main analytic object of the method is the complex momentum $p$ defined by the formula

$$
2 \cos p+v(z)=0 .
$$

It is a multivalued analytic function. The branch points of $p$ are called turning points. At turning points, one has $\cos p(z) \in\{ \pm 1\}$ and $v(z) \in\{ \pm 2\}$.

We call a set $D \subset U$ regular if $v(z) \neq \pm 2$ in $D$.

\subsection{The main theorem of the complex WKB method}

As in the case of differential equations, the main geometric notion of the complex WKB method is the one of canonical domain. The reader can find definitions and details in $[9,10]$. Here, we note only that canonical domains are regular simply connected domains independent of $h$. The following two theorems hold.

Theorem 1 Any regular point belongs to a canonical domain.

This statement is essentially Lemma 5.2 from [11].

Theorem 2 ([9, 10]) Let $K \subset U$ be a canonical domain, $z_{0} \in K$, and $p$ be a branch of the complex 
momentum analytic in $K$. Suppose that either $K$ is a bounded canonical domain or $v$ is a trigonometric polynomial. Then, there exist solutions $\psi_{ \pm}$to (1) analytic in $K$ and such that as $h \rightarrow 0$

$$
\psi_{ \pm}(z)=\frac{\exp \left( \pm \frac{i}{h} \int_{z_{0}}^{z} p(z) d z+o(1)\right)}{\sqrt{\sin (p(z))}}, z \in K .
$$

This asymptotic representation is locally uniform.

We note that, at a branch point, one has $\cos p(z) \in$ $\{ \pm 1\}$, and so, $\sin p(z)=0$. Therefore, formula (2) can not be valid near a branch point.

For the equation $-h^{2} \psi^{\prime \prime}(z)+v(z) \psi(z)=$ 0 , formula $(2)$ is replaced with $\psi_{ \pm}(z)=$ $\frac{1}{\sqrt{p(z)}} e^{ \pm \frac{i}{h} \int_{z_{0}}^{z} p(z) d z+o(1)}$, where the complex momentum is defined by the relation $p^{2}+v(z)=0$, i.e., as for (1), by the symbol of the equation.

\section{A WARM UP}

Here, we define objects and recall some facts needed to formulate our results.

\subsection{Complex momentum near a branch point}

Below, $z_{0} \in U$ is a branch point of the complex momentum $p$.

We note that as $\cos \left(p\left(z_{0}\right)\right) \in\{ \pm 1\}$, one has either $p\left(z_{0}\right)=0 \bmod 2 \pi$ or $p\left(z_{0}\right)=\pi \bmod 2 \pi$.

If $v^{\prime}\left(z_{0}\right) \neq 0, p$ is analytic in $\tau=\sqrt{z-z_{0}}$ in a neighborhood of zero, and

$$
p(z)=p\left(z_{0}\right)+k_{1} \tau+\ldots, \quad \tau \rightarrow 0, \quad k_{1} \neq 0 .
$$

In this case, the branch point $z_{0}$ is called simple.

In this paper we assume that

- in $U$, there is a single branch point $z_{0}$ of the complex momentum and it is simple; for the sake of simplicity, we assume that $U$ is a disk, and that $z_{0}$ is its center;

- $p\left(z_{0}\right)=0 \bmod 2 \pi$.

Note that, the case $p\left(z_{0}\right)=\pi \bmod 2 \pi$ can obtained by a simple algebraic transformation from the case $p\left(z_{0}\right)=0 \bmod 2 \pi$.

\subsection{Function $\zeta$}

We cut $U$ from $z_{0}$ along, say, the line $\left\{z-z_{0} \leq 0\right\}$ and denote the thus obtained domain by $U^{\prime}$. In $U^{\prime}$, we fix $p$, an analytic branch of the complex momentum and $\zeta$, an analytic branch of the function

$$
z \mapsto\left(\frac{3}{2 i} \int_{z_{0}}^{z}\left(p(z)-p\left(z_{0}\right)\right) d z\right)^{\frac{2}{3}}
$$

The function $\zeta$ is analytic in $U$. One has $\zeta\left(z_{0}\right)=0$, and $\zeta^{\prime}\left(z_{0}\right) \neq 0$. Possibly reducing $U$ somewhat, we can and do assume that

- $\zeta$ is analytic isomorphism of $U$ onto its image.

\subsection{Basic facts on Airy functions}

The equation

$$
w^{\prime \prime}(\zeta)=\zeta w(\zeta), \quad \zeta \in \mathbb{C},
$$

is the Airy equation. Its solutions are called Airy functions.

Let $|\arg z|<2 \pi / 3$. There exists an Airy function Ai that admits the following asymptotic representations as $|z| \rightarrow \infty$ (see [12])

$$
\begin{gathered}
\operatorname{Ai}(z)=\frac{\exp \left(-\frac{2}{3} z^{\frac{3}{2}}+o(1)\right)}{2 \sqrt{\pi} z^{\frac{1}{4}}}, \\
\operatorname{Ai}(-z)=\frac{\cos \left(\frac{2}{3} z^{\frac{3}{2}}-\frac{\pi}{4}+o(1)\right)}{\sqrt{\pi} z^{\frac{1}{4}}}(1+o(1)),
\end{gathered}
$$

where we use the analytic branches of $z \rightarrow z^{\frac{3}{2}}$ and $z \rightarrow z^{\frac{1}{4}}$ that are positive for $z>0$.

Let $\omega=e^{2 \pi i / 3}$. For $j \in \mathbb{Z}_{3}$, we define $w_{j}(\cdot)=$ $\operatorname{Ai}\left(\omega^{j} \cdot\right)$. The functions $\left(w_{j}\right)_{j \in\{1,2\}}$ are two more Airy functions.

\section{Analytic solutions in a COMPlex Neigh- BORHOOD OF A BRANCH POINT}

\subsection{Asymptotic solutions}

For a function $f$ defined in $U$ and a point $z \in U$ s.t. $z \pm h \in U$, we set

$$
H f(z)=f(z+h)+f(z-h)+v(z) f(z) .
$$

We let

$$
g(z):=\frac{\sinh \left(\sqrt{\zeta(z)} \zeta^{\prime}(z)\right)}{\sqrt{\zeta(z)}}, \quad z \in U,
$$

where the determination of the square roots in the denominator and the numerator are the same. The definition of $g$ is independent of the choice of this 
determination, and $g$ is analytic and does not vanish in $U$. We further denote by $A_{0}$ a branch of the function $z \rightarrow \frac{1}{\sqrt{g(z)}}$ that is analytic in $U$. One has

Theorem 3 There exist $\left(A_{l}\right)_{l \in \mathbb{N}}$ and $\left(B_{l}\right)_{l \in \mathbb{N}}$, analytic functions on $U$, such that the following holds. Let $w$ be one of the Airy functions $\left\{w_{j} ; j \in \mathbb{Z}_{3}\right\}$. Pick $L \in \mathbb{N} \cup\{0\}$ and put

$$
\begin{aligned}
& W(z)=h^{\frac{1}{3}} w \sum_{l=0}^{L} h^{l} A_{l}(z)+h^{\frac{2}{3}} w^{\prime} \sum_{l=1}^{L} h^{l} B_{l}(z), \\
& w=w\left(\zeta(z) / h^{\frac{2}{3}}\right), \quad w^{\prime}=w^{\prime}\left(\zeta(z) / h^{\frac{2}{3}}\right) .
\end{aligned}
$$

One has the locally uniform estimates

$$
H(W)=O\left(h^{L+2+\frac{1}{3}} w\right)+O\left(h^{L+2+\frac{2}{3}} w^{\prime}\right) .
$$

The coefficient $A_{0}$ was defined just above Theorem 3. There are simple induction formulas for the other coefficients.

We call the formal expression

$$
h^{\frac{1}{3}} w \sum_{l=0}^{\infty} h^{l} A_{l}(z)+h^{\frac{2}{3}} w^{\prime} \sum_{l=1}^{\infty} h^{l} B_{l}(z)
$$

an asymptotic solution to (1).

For the differential equation $-\psi^{\prime \prime}(z)+v(z) \psi(z)=$ 0 , in a neighborhood of a simple turning point (a point where $v(z)=0$ and $v^{\prime}(z) \neq 0$ ), there are asymptotic solutions of the form (5) (with different coefficients $A_{l}, B_{l}$ and a different function $\zeta$ ), see, e.g., [6, 7]. To justify the Ansatz (5) in the case of the difference equation requires to develop original techniques. In particular, one has to derive asymptotic formulas of the form

$$
\begin{aligned}
w\left(h^{-\frac{2}{3}} \zeta(z+h)\right) & =h^{\frac{1}{3}} f(z) w\left(h^{-\frac{2}{3}} \zeta(z)\right) \\
& +h^{\frac{2}{3}} g(z) w^{\prime}\left(h^{-\frac{2}{3}} \zeta(z)\right)+\ldots
\end{aligned}
$$

where $f(z)=\cosh \left(\sqrt{\zeta(z)} \zeta^{\prime}(z)\right)$ and the dots denote smaller order terms. Trying to obtain this formula using Taylor expansions for the left hand side leads to a series containing an infinite number of infinite subsequences of terms of same order. So, one has to find an effective way to resum these sequences. To get formulas similar to (6), instead of resumming Taylor series, we use tools of the complex analysis.

\subsubsection{Exact solutions}

Let us turn to exact analytic solutions to (1). The main result of this note is
Theorem 4 There exists $\stackrel{\circ}{U} \subset U$ a neighborhood of $z_{0}$ independent of $h$ such that the following holds. Pick $L \in \mathbb{N}$ and $w \in\left\{w_{j} ; j \in \mathbb{Z}_{3}\right\}$. Let $W$ be the function constructed in Theorem 3 for these $L$ and $w$. For sufficiently small $h$, there exists a solution $\psi$ to equation (1) that is analytic in $\stackrel{\circ}{U}$ and, in $\stackrel{\circ}{U}$, admits the uniform asymptotic representation

$$
\psi(z)=W(z)+O\left(h^{L+1+\frac{1}{3}} w\right)+O\left(h^{L+1+\frac{2}{3}} w^{\prime}\right),
$$

where $w$ and $w^{\prime}$ are defined in (4).

Let us briefly explain the idea of the proof of this result. First, using the approximate solutions from Theorem 3, we construct a parametrix $R$, i.e., an operator such that, for suitable functions $f$, one has $H R f=f+D f$, where $H$ is defined in (3), and $D$ is a small operator. The operator $D$ appears to be a singular integral operator. To estimate its norm, one has to deal with geometric objects natural for complex WKB method. This allows to prove Theorem 4 for some special subdomains of $U$. Then, one studies the thus constructed solutions in larger domains.

We note that, as equation (1) is non-local in $z$, the ideas underlying the analysis of (1) are quite different from the ideas used to get similar results for differential equations (see, e.g., $[6,7]$ ).

\subsection{Related results}

In [13] and [14] the authors essentially studied equations of the form

$$
Y_{k+1}=M(h k) Y_{k}, \quad k \in \mathbb{Z},
$$

with a small positive $h$ and a $(n \times n)$-matrix function $M$ defined on $\mathbb{R}$. If

$$
Y(x+h)=M(x) Y(x), \quad x \in \mathbb{R}
$$

then the sequence $Y_{k}=Y(k h), k \in \mathbb{Z}$, satisfies (7). Equation (1) restricted to $\mathbb{R}$ is equivalent to (8) with $M(x)=\left(\begin{array}{cc}-v(x) & -1 \\ 1 & 0\end{array}\right)$, and the points $x$ where the two eigenvalues of this matrix coalesce are branch points of the complex momentum.

The short note [13] is mainly devoted to the case when all the eigenvalues of the matrix $M$ in (7) are distinct. In [14] the author constructed asymptotic solutions to (7) in a small as $h \rightarrow 0$ neighborhood of a point where two eigenvalues of $M(x)$ coincide. 
In [15], the authors considered the equations

$$
\sum_{j=0}^{J} a_{j}(h k, h) y_{k+j}=0, \quad k \in \mathbb{Z} .
$$

We note that this class includes the difference Schrödinger equations

$$
y_{k+1}+y_{k-1}+v(h k) y_{k}=0, \quad k \in \mathbb{Z} .
$$

The authors described the asymptotics of solutions to (9) for $h k$ in a small (as $h \rightarrow 0$ ) neighborhood of a point where $v(x) \in\{ \pm 1\}$.

There is a series of papers by J.S. Geronimo and coauthors, see, e.g. [5] and references therein, devoted to uniform asymptotic formulas for solutions to the equation $a_{k+1} \psi_{k+1}+b_{k} \psi_{k}+a_{k} \psi_{k-1}=x \psi_{k}$, $k \in \mathbb{Z}$, where $x$ is the spectral parameter, the coefficients $\left(a_{n}\right)_{n}$ are positive and $\left(b_{n}\right)_{n}$ are real.

We also mention paper [16] where the authors constructed approximate solutions to a difference equation using the Maslov canonical operator.

\section{ACKNOWLEDGMEnTs}

This work was supported by the CNRS and the Russian foundation of basic research under the French-Russian grant 17-51-150008.

The authors are grateful to Evgenii Vybornyi for the references to papers $[13,14]$ and to Sergei Dobrokhotov for [16].

\section{REFERENCES}

[1] Fedotov, A.,A., 2014, Monodromization method in the theory of almost-periodic equations, St. Petersburg Mathematical Journal, Vol. 25, pp. 303-325.

[2] Helffer, B., Sjöstrand, J., 1988, Analyse semiclassique pour l'equation de Harper (avec application à l'étude de l'équation de Schrödinger avec champ magnétique), Mémoires de la $S M F$, Vol. 34, pp. 1-113.

[3] Babich, V., Lyalinov, M., Grikurov, V., 2008, Diffraction theory: the SommerfeldMalyuzhinets technique, Alpha Science, Oxford.

[4] Fedotov, A., Klopp, F., 2002, Anderson transitions for a family of almost periodic Schrödinger equations in the adiabatic case, Communications in Mathematical Physics, Vol. 227, pp. 192.
[5] Geronimo, J., S., Bruno, O., Van Assche, W., 1992, WKB and Turning Point Theory for Second-order Difference Equations, Operator theory: advances and app., Vol. 69, pp. 269301.

[6] Fedoryuk, M., V., 2009, Asymptotic analysis. Linear ordinary differential equations, SpringerVerlag, Berlin, Heidelberg.

[7] Wasow, W., 1987, Asymptotic expansions for ordinary differential equations, Dover Publications, New York.

[8] Buslaev, V., Fedotov, A., 1995, Complex WKB method for Harper equation, St. Petersburg Math. J., Vol. 6, pp. 495-517.

[9] Fedotov, A., Shchetka, E., 2015, The complex WKB method for difference equations in bounded domains, J. of Math. Sciences (New York), Vol. 438, pp. 236-254.

[10] Fedotov, A., Shchetka, E., 2018, Complex WKB method for the difference Schrödinger equation with the potential being a trigonometric polynomial, St. Petersburg Mathematical Journal, Vol. 29, pp. 363-381.

[11] Fedotov, A., Klopp, F., 2005, On the absolutely continuous spectrum of an onedimensional quasi-periodic Schrödinger operator in adiabatic limit, Transactions of $A M S$, Vol. 357, pp. 4481-4516.

[12] Olver, F., W., J., 1974, Asymptotics and Special Functions, Academic Press, New York.

[13] Vasilieva, A., B., Yuldashev, E., 1970, Linear difference systems with small lag, Diff. equations (in Russian), Vol. 62, pp. 267-269.

[14] Tsyganov, G., A., 1974, Asymptotic behavior of solutions of a linear difference system with small difference in the presence of a turning point, Diff. equations (in Russian), Vol. 10, pp. 1312-1321.

[15] Costin, O., Costin, R., 1996, Rigorous WKB for finite-order linear recurrence relations with smooth coefficients, SIAM J. math. an., Vol. 27, pp. 110-134.

[16] Dobrokhotov, S., Yu., Tsvetkova, A., V., On lagrangian manifolds related to asymptotics of Hermite polynomials, Math. notes, Vol. 110, to appear. 